\title{
A Computer Model of the Spread of the Pandemic and its Analysis
}

\author{
Avtandil Bradvelidze \\ Department of Computer \\ Technology \\ Akaki Tsereteli State University \\ Kutaisi, Georgia \\ Email: bardaveli54 [AT] mail.ru
}

\author{
Irakli Basheleishvili \\ Department of Computer \\ Technology \\ Akaki Tsereteli State University \\ Kutaisi, Georgia \\ Email: basheleishvili.irakli [AT] \\ gmail.com
}

\author{
Khatuna Bradvelidze \\ Department of Informatic \\ Georgian Technical University \\ Tbilisi, Georgia \\ Email: bardaveli [AT] yandex.ru
}

\begin{abstract}
The paper describes and analyzes a mathematical model of the variable state of the incidence of epidemic diseases, which is of great importance for determining the quantity of vaccines and antiviral drugs to be produced. The information model according to the system of differential equations of the spread of the pandemic is illustrated in a structural diagram. The model is presented in a vector-matrix form and the state of equilibrium of the model in the spatial state is proved.The model of the spread of the pandemic was developed, whose implementation with a Matlab software package resulted in obtaining the curves of variation of the state. The developed computer model of the incidence of epidemic diseases can be used to make a projection of the number of infected people, as well as intensity of the process of disseminating information and ideas in the community.
\end{abstract}

Keywords- Pandemic; Computer Model; Matlab; Analysis.

\section{INTRODUCTION}

Environmental changes, global warming, rising population density, high migration activity and other factors are leading to the emergence and spread of new infections in the world.

The emergency caused by a new coronavirus (COVID-19) is an integral part of the universe, so mankind should learn to confront these challenges. A strict study of this problem can only be started on the basis of a properly formulated mathematical model.

The emergence of coronavirus (COVID-19) diseases in December 2019 became known as an emergency situation of international concern. It is well known that in the new millennium, mankind faced an infectious disease of which little is known.

Obviously, infections have no boundaries. According to projections of the United Nations, the world population will continue to grow to 10 billion by the year 2050 . This means that the migration and urbanization processes will be further accelerated. The COVID-19 pandemic went down in history as an emergency situation of international concern. It is clear that the emergence of new viruses is an integral part of our world. Mankind should learn to withstand these threats.
A thorough study of all aspects of this problem can only be started on the basis of the well-formulated mathematical models.

In order to determine the number of registered cases, the number of patients admitted into the hospital, and the number of fatal cases, it is undoubtedly necessary to develop and analyze a mathematical model of the incidence of epidemic diseases, which will also help all countries to develop and take emergency measures. Special importance is attached to a mathematical model, which will be developed for determining the number of vaccines and antiviral drugs to be produced $[1,2]$.

\section{BASIC PART}

The number of people to be screened can be divided into three groups $x_{1}, x_{2}$ and $x_{3}$, where $x_{1}$ is group of people vulnerable to the epidemic diseases, $x_{2}$ - a group of infected people and $x_{3}$ - a group of people excluded from the initial number of people to be screened. Exclusion of the group $x_{3}$ is made depending on the causes of immunization, fatality or isolation from the group $x_{1}$. The incidence of epidemic diseases can be described by the system of differential equations, which includes feedback and has the form as follows $[3,4]$ :

$$
\begin{aligned}
& \dot{x}_{1}=-\alpha x_{1}-\beta x_{2}+u_{1}(t) \\
& \dot{x}_{2}=\beta x_{1}-\gamma x_{2}+u_{2}(t) \\
& \dot{x}_{1}=-\alpha x_{1}+\gamma x_{2}
\end{aligned}
$$

The speed at which new sensitivity to the disease is emerged is equal to $u_{1}(t)$; the speed at which new introduction of infection occurs is equal to $u_{2}(t)$. In the isolated community, $u_{1}(t)=u_{2}(t)=0 . \alpha, \beta, \gamma$ - are the positive proportionality factors that characterize intensity of 
the decrease or completion of the groups.

information flow in the model.

The structural diagram (Fig. 1) illustrates the

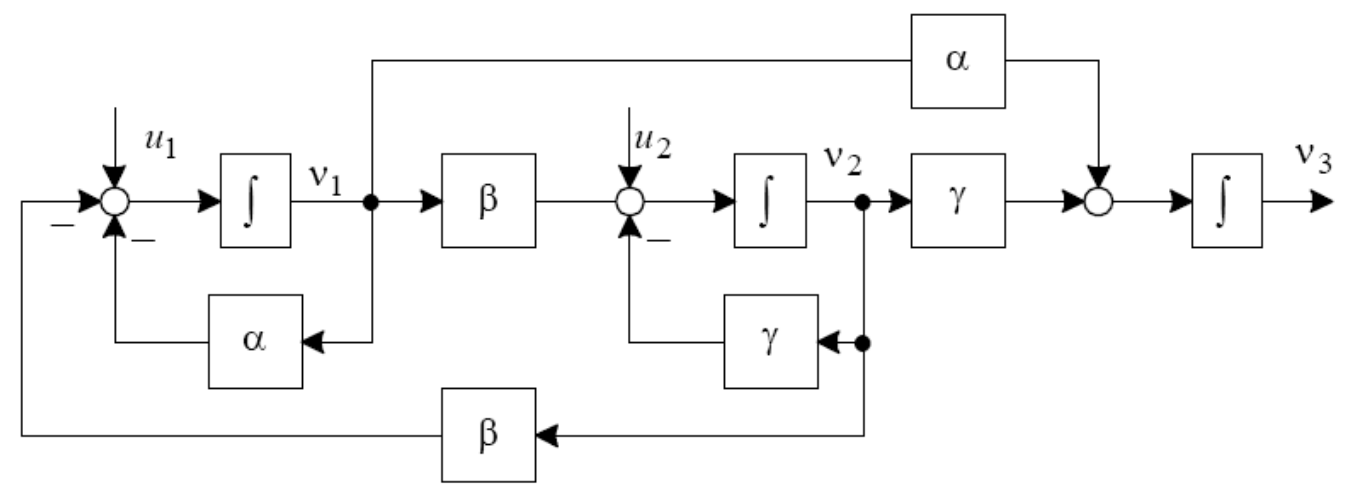

Figure 1. The structural scheme of modeling the incidence of epidemic diseases

In a system (1), $x_{1}, x_{2}$ and $x_{3}$ are the physically variable states. The equation system can be presented in the vector-matrix form:

$$
\dot{\mathbf{X}}=\mathbf{A} x+\mathbf{B} u
$$

where

$$
\mathbf{A}=\left[\begin{array}{ccc}
-\alpha & -\beta & 0 \\
\beta & -\gamma & 0 \\
\alpha & \gamma & 0
\end{array}\right], \quad \mathbf{B}=\left[\begin{array}{ll}
1 & 0 \\
0 & 1 \\
0 & 0
\end{array}\right]
$$

The analysis of the equation (2) reveals that the variable state $x_{3}$ depends on $x_{1}$ and $x_{2}$, however it does not have influence on them. Let us consider the isolated community, which $u_{1}(t)=u_{2}(t)=0$. In a spatial state, it is consistent with a state of equilibrium $\dot{x}=0$. The analysis of the equation (2) reveals that the system is in a state of equilibrium, when $x_{1}=x_{2}=0$. In order to establish whether the incidence of epidemic disease is stopped, it is necessary to consider the polynomial characterizing $\operatorname{det}(s E-A)$. At this time, we should take into consideration that the third equation in a system (1) does not have influence on the first and second equations (the third equation is not indicated in Figure 1 in any contour), thus it can be excluded from the analysis. Then, to study the initial conditions, the model will have the vector form [3]:

$$
\dot{\tilde{\mathbf{X}}}=\tilde{\mathbf{A}} \tilde{\mathbf{X}}
$$

where

$$
\tilde{\mathbf{X}}=\left[\begin{array}{l}
x_{1} \\
x_{2}
\end{array}\right], \tilde{\mathbf{A}}=\left[\begin{array}{c}
-\alpha-\beta \\
\beta-\gamma
\end{array}\right]
$$

On the assumption that $\alpha=\gamma=\beta=1$, then the characteristic equation will have the following form:

$$
S^{2}+2 S+2=0 \text {, }
$$

whose roots are complex. Since $\alpha+\gamma>0, \alpha \gamma+\beta^{2}>0$, the roots of the characteristic equation are placed in the left half-plane of the complex plane. Proceeding from this assumption, the free movement asymptomatically tends to zero, that is

$$
\mathbf{X}_{\mathrm{Tav}}(t) \rightarrow 0
$$

and the $\tilde{\mathbf{X}}=0$ derivative state will be a steady state. 


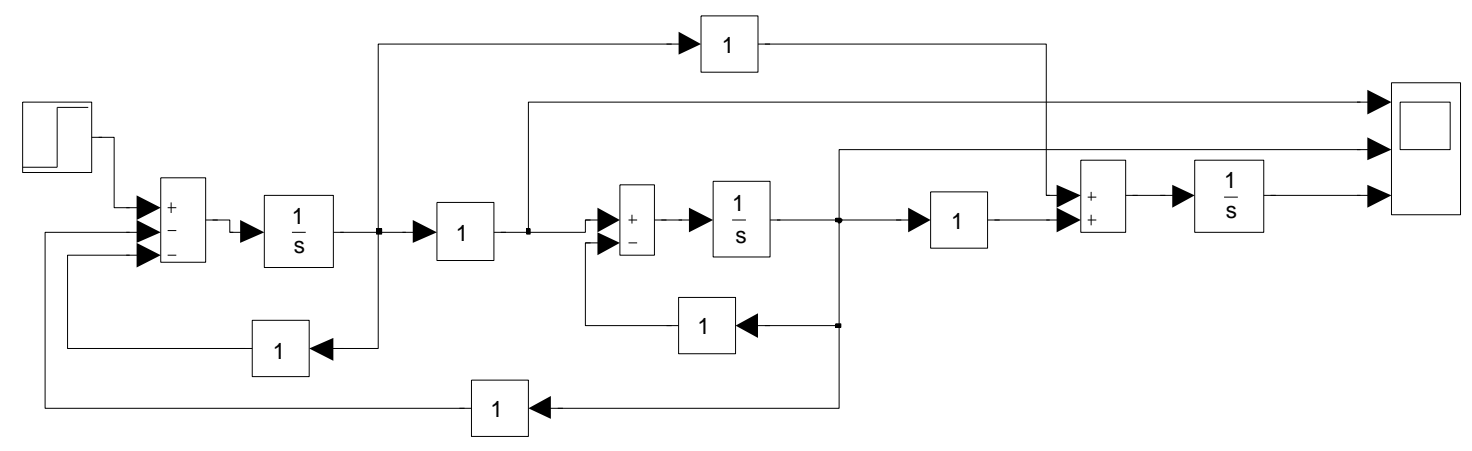

Figure 2. A computer model of the incidence of epidmic disease

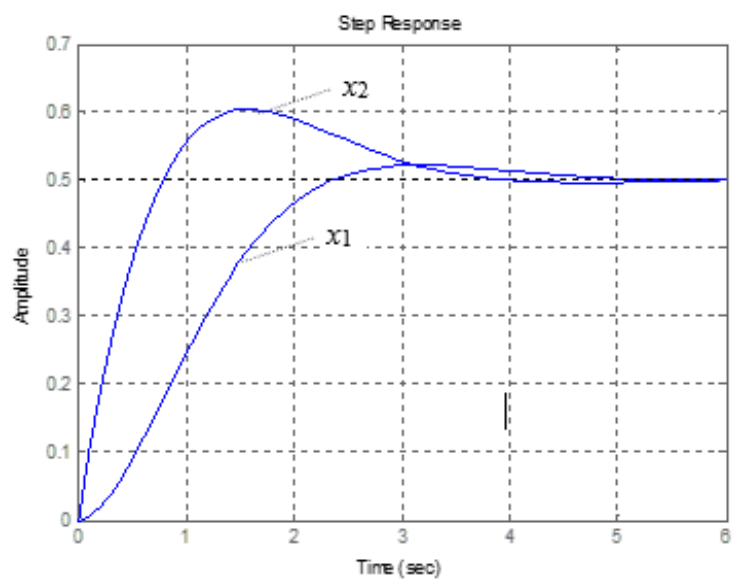

Figure 3. The ransient characteristics of the incidence of the epidemic diseases

The result of the above mentioned analysis is illustrated by means of a computer block model of modeling the structural diagram shown in Figure 1 (Fig. 2), whose implementation with a Matlab software package (4) resulted in obtaining the curves of variables of the states $x_{1}$ and $x_{2}$.

\section{CONCLUSION}

The developed computer model of the incidence of epidemic diseases allows to make a projection of the number of infected people, as well as intensity of the process of disseminating information and ideas in the community.

\section{REFERENCES}

[1] Coronavirus COVID-19 Global Cases by the Center for Systems Science and Engineering (CSSE) at Johns Hopkins University. March 21, 2020.

[2] Guan W. J. et coll. (2020) Clinical characteristics of 2019 novel coronavirus infection in China. New England Journal of Medicine, doi : 10.1056/NEJMoa2002032

[3] S.E. Dushin, A.V. Krasov, Yu.V. Litvinov. Modeling of the systems and complexes. The training manual/ St. Petersburg: SPBGU ITMO, 2010. $177 \mathrm{p}$.

[4] Bardavelidze, K., \& Bardavelidze, A. Simulation Modeling of a Queuing System in the MATLAB Environment.

[5] Bardavelidze, K., \& Bardavelidze, A. (2012, October). Imitation modeling of compatitive market equilibrium. In 2012 6th International Conference on Application of Information and Communication Technologies (AICT) (pp. 1-4). IEEE.

[6] J. Clerk Maxwell, A Treatise on Electricity and Magnetism, 3rd ed., vol. 2 